\title{
Breeding of new disease-tolerant grape varieties - Viticulture in times of climatic change
}

\author{
E. Weinmann, M. Boos, P. Ehret, L. Flubacher, C. Schneider, and L. Veith \\ State Institute of Viticulture and Oenology, 79100 Freiburg, Germany
}

\begin{abstract}
The State Institute of Viticulture and Oenology (WBI) Freiburg Germany has been breeding disease-tolerant grape varieties (Piwis) since the 1930s. Since that time, 15 fungus-resistant white and red wine varieties and 4 table grape varieties have been cultivated. All these grape varieties are approved as Vitis vinifera. Until now, fungus resistance has been the focus of breeding work. Due to climate changings, it will be necessary in future to delay budding and ripening of grapevines. This would protect the vines from late frosts in spring and delay the ripening of the berries in autumn. In addition, the loosening of the grape structure and dry resistance will be future breeding goals. To improve the resistance and a later ripeness of the berries, new grape varieties which are currently being tested have been developed in cooperation with the INRA in Colmar (France).
\end{abstract}

\section{Einleitung}

Seit der Einschleppung des Falschen Mehltaus (Peronospora) und des Echten Mehltaus (Oidium) aus Amerika im 19. Jahrhundert sind hohe Ertrags- und Qualitätseinbußen beim Anbau von traditionellen Europäerrebsorten nur durch den Einsatz von Pflanzenschutzmitteln $\mathrm{zu}$ verhindern. Um den Einsatz von Pflanzenschutzmitteln zu minimieren, beschäftigt sich das WBI seit den 1930er Jahren damit, die Resistenz amerikanischer und asiatischer Reben mit der Qualität der Europäersorten zu kombinieren. Dabei ist es durch die Nutzung ansprechender Europäer- Amerikaner-Hybriden, verschiedener Wildarten, pilzwiderstandsfähiger osteuropäischer und asiatischer Zuchtstämme und durch die gezielte Rückkreuzung mit Europäerreben gelungen, die Weinqualität der neuen pilzwiderstandsfähigen Rebsorten auf das Niveau der traditionellen Qualitätsweinsorten zu bringen. Aus diesen Kreuzungen sind in den 1970er und 1980er Jahren 15 pilzwiderstandsfähige Weiß- und Rotweinsorten entstanden, die sich derzeit im Anbau befinden. Diese Rebsorten sind auf Grundlage der UPOV Merkmale kategorisiert und als Vitis vinifera Rebsorten zugelassen. Die Anbaufläche dieser Rebsorten hat sich in den letzten Jahren auch international positiv entwickelt. Bei diesen Rebsorten ist es nun notwendig, die oenologischen Untersuchungen weiterzuführen. Den Winzerinnen und Winzern soll so ein zielgerichteter Ausbau der Weine ermöglicht werden, um auf dem Weinmarkt erfolgreich zu sein.

Die Klimaentwicklung in den letzten Jahrzehnten hat in Südwestdeutschland dazu geführt, dass bei den Burgundersorten das Rebenentwicklungsstadium Knospenaufbruch durchschnittlich 13 Tage früher, das Stadium Vollblüte durchschnittlich 21 Tage früher und der Lesebeginn durchschnittlich 15 Tage früher erreicht werden. Aufgrund der Veränderung der Temperaturverläufe entstand in den letzten beiden Jahrzehnten eine intensive Diskussion über die Pflanzung internationaler Rebsorten.

Aufgrund dieser Klimaentwicklung spielt, neben der Pilzwiderstandsfähigkeit und Traubenstruktur, auch die Züchtung von Sorten mit einer verzögerten Entwicklung eine besondere Rolle. Aus diesem Grund wurden in den Jahren 2004 bis 2006 Kreuzungen mit dem Vitis Muscadinia Genpool durchgeführt, um eine Pyramidisierung der Resistenzgene zu erzielen. Zur Erstellung dieser Kreuzungspartner wurden unter anderem Rückkreuzungen mit den Rebsorten Cabernet Sauvignon, Grenache, Merlot und Aubun durchgeführt [1,2]. Die hieraus entstandenen Nachkommen werden in den nächsten Jahren weiter auf ihre weinbaulichen und oenologischen Eigenschaften überprüft.

\section{Auswirkungen der klimatischen Entwicklung auf die Rebenentwicklung in Südwestdeutschland}

Die Auswirkungen der klimatischen Entwicklung in Südwestdeutschland soll am Beispiel der Entwicklungsstadien Knospenaufbruch, Vollblüte und Lesebeginn der Burgundersorten im Markgräflerland seit dem Jahr 1976 [3] aufgezeigt werden.

Der Knospenaufbruch hat sich in diesem Zeitraum durchschnittlich um 13 Tage verfrüht. Dabei fällt auf, dass die Unterschiede in den einzelnen Jahren zwar geringer werden, die Schwankungen seit dem Jahr 2000 aber immer noch bis zu 22 Tagen betragen haben. Durch die Verfrühung des Austriebs steigt in den deutschen Anbaugebieten auch die Gefahr von Schädigungen durch Spätfrost. In den Jahren 2014 und 2019 kam es nur zu leichten Frostschäden, die keinen Einfluss auf den Ertrag hatten. Spätfröste, wie im Jahr 2017, haben aber vor Augen 


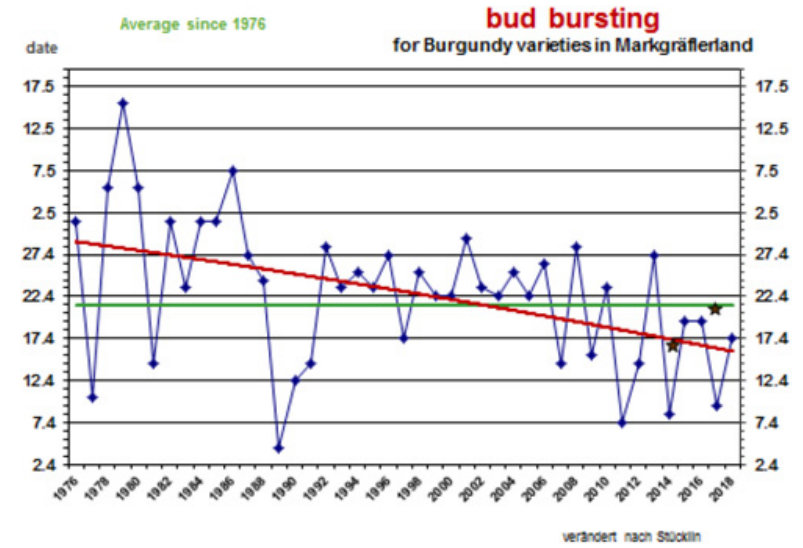

Abb 1. Entwicklung des Austriebstermins im Markgräflerland seit 1976.

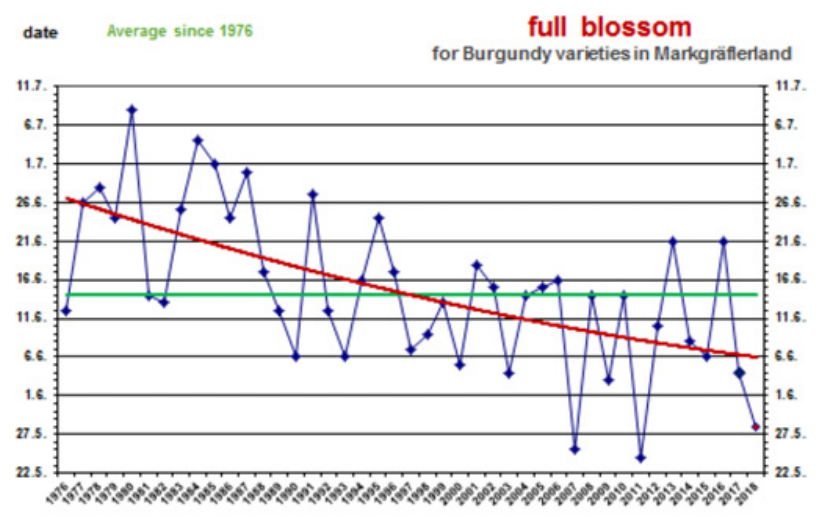

verasoen nach swockin

Abb 2. Entwicklung des Blütetermins im Markgräflerland seit 1976.

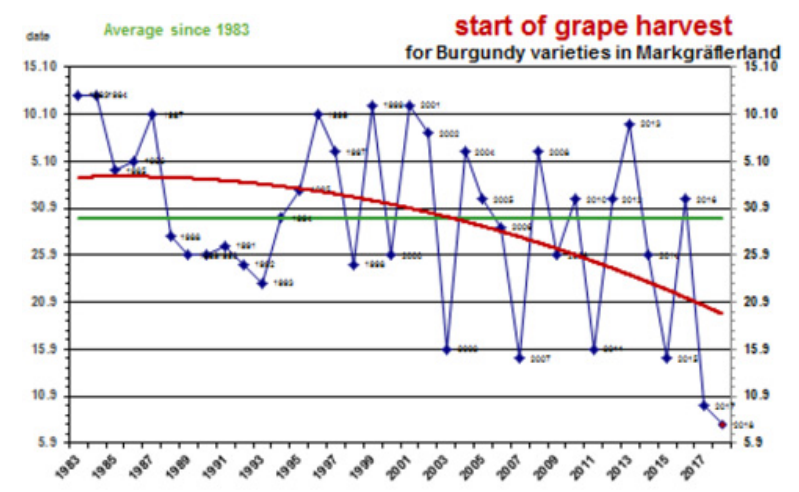

veranoent naon swowin

Abb 3. Entwicklung des Lesebeginns von Burgundersorten im Markgräflerland seit 1983.

geführt, dass diese ein nicht zu unterschätzendes Risiko für den Weinbau in Deutschland sein können.

Die Vollblüte hat sich in diesem Zeitraum durchschnittlich um 21 Tage verfrüht. Auch hier fallen die immensen Schwankungen in den unterschiedlichen Jahren auf. In den Jahren seit 2000 sind Schwankungen von bis zu vier Wochen festzustellen.

Der Lesebeginn hat sich in den letzten drei Jahrzehnten durchschnittlich um 15 Tage verfrüht. Es fallen die sehr unterschiedlichen Lesetermine auf, die in den Jahren 1999 und 2018 um 44 Tage differieren. Aus diesen sehr unterschiedlichen Jahren entstehen große

\section{Aims of the resistant breeding of State Institute of Viticulture and Oenology}

\author{
- Aims of breeding \\ - breeding tolerant varieties \\ - loose grape structure \\ - delay of the vegetation \\ - dry resistance \\ - upright growth \\ - wine quality \\ - market
}

Abb 4. Ziele der Resistenzzüchtung des WBI.

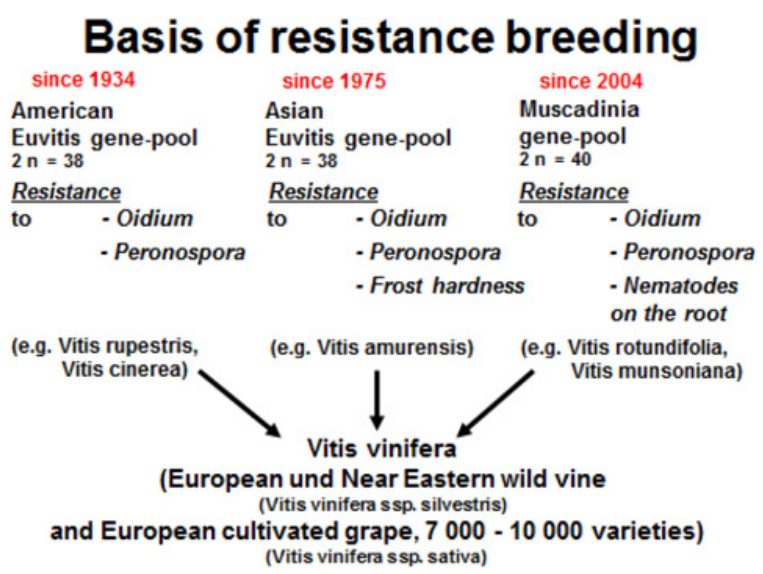

Abb 5. Basis der Resistenzzüchtung des WBI.

Herausforderungen für die Oenologen, die sortentypischen Aromen vor allem der Weißweine zu erhalten.

\section{Ziele der Rebenzüchtung am WBI}

Für das WBI ergibt sich für die Züchtungsarbeit, neben der Pilzwiderstandsfähigkeit und der Lockerbeerigkeit, als weiteres Zuchtziel neue Sorten mit späterem Austriebsund Traubenlesetermin zu züchten. Darüber hinaus ist es notwendig, neue Rebsorten auszulesen, die bezüglich weiterer weinbaulicher Parameter, wie beispielsweise den aufrechten Wuchs und die Rankfähigkeit, Vorteile für den Winzer bringen. Die Weinqualität ist aber sicherlich der ausschlaggebende Faktor, der darüber entscheidet, ob die neuen Rebsorten an die Winzerinnen und Winzer weitergegeben werden. Weiterhin ist es Ziel des WBI, die Vermarktung der Weine aus den neuen pilzwiderstandsfähigen Rebsorten zu verbessern und den Winzern Informationen für eine effiziente Vermarktungsstrategie mit auf den Weg zu geben.

\section{Können internationale Rebsorten zur Lösung des Problems beitragen?}

Vor dem Hintergrund der Klimaerwärmung wurde in Deutschland in den letzten beiden Jahrzehnten immer wieder der Anbau von mediterranen Rebsorten diskutiert. Um zu prüfen, ob ein Wechsel auf Rotweinsorten, die traditionell in südlich mediterranen Anbaugebieten beheimatet sind, zur nachhaltigen Erzeugung von verkaufsfähigen Weinqualitäten möglich ist, wurden am WBI in den Jahren 1998 bis 2008 Untersuchungen mit 


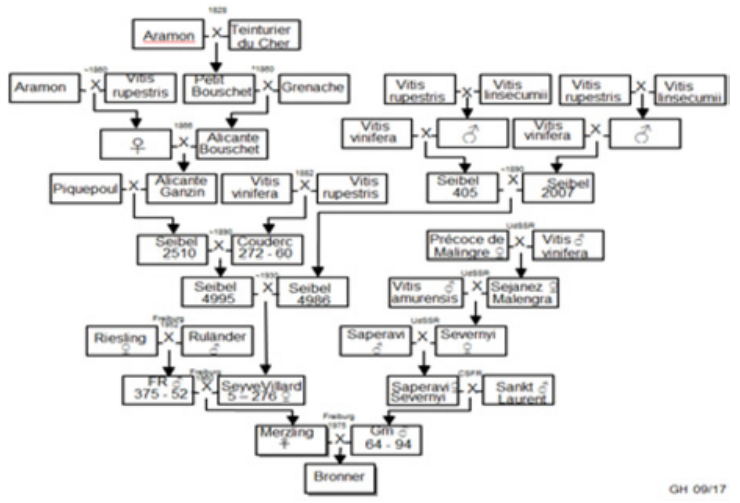

Abb 6. Stammbaum der Rebsorte Bronner.

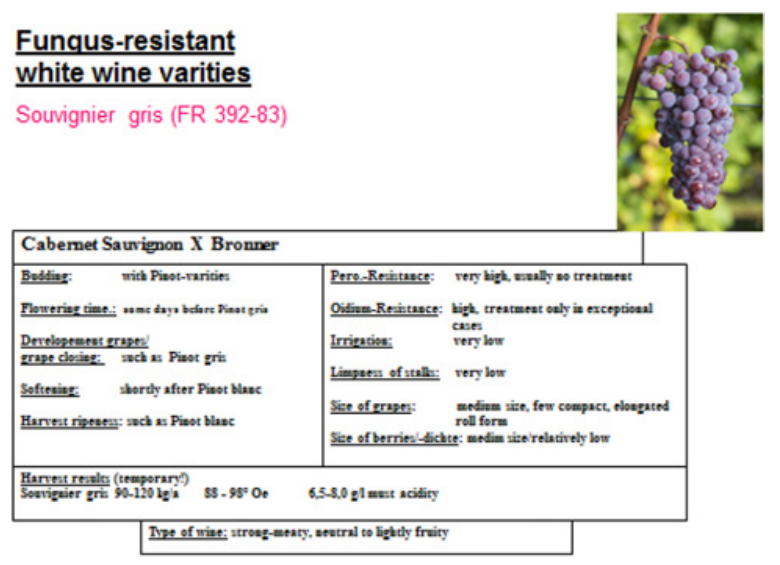

Abb 7. Aufstellung über die phänologische Daten, die Resistenzeigenschaften und Beschreibung des Weintyps der Rebsorte Souvignier Gris.

verschiedenen Rebsorten aus südlichen Anbaugebieten durchgeführt. Dabei konnte festgestellt werden, dass die Mostgewichtssteigerungen während der Reifephase des Spätburgunders im Wesentlichen von der Rebsorte Merlot erreicht wurden, während Cabernet Sauvignon, Barbera und insbesondere Nebbiolo und Sangiovese um 10 bis 20 Grad Oechsle hinter dem, jeweils höchsten, vom Spätburgunder erreichten Mostgewicht zurückbleiben [4].

Der Verlauf der Mostsäureentwicklung für die entsprechenden Sorten zeigt, dass einstellige Mostsäurewerte zum Lesetermin regelmäßig nur von den Rebsorten Spätburgunder, Merlot und Cabernet Sauvignon erreicht werden [4].

Daraus ergibt sich, dass Rebsorten aus den südlichen Anbaugebieten nicht zur Lösung des Problems beitragen können.

\section{Ergebnisse der Rebenzüchtung des WBI}

Das WBI betreibt seit dem Jahr 1934 die Züchtung pilzwiderstandsfähiger Rebsorten. Zu Beginn der Arbeiten wurden Kreuzungen mit Vitis rupestris und Vitis cinerea durchgeführt. Im Jahr 1975 wurden Zuchtstämme mit dem Resistenzpartner Vitis amurensis in das Kreuzungsprogramm aufgenommen. In dieser Phase stand, neben der Pilzwiderstandsfähigkeit, auch die Weinqualität im Vordergrund der Arbeiten.

Aus diesen Kreuzungen sind in den 1970er und 1980er Jahren 15 pilzwiderstandsfähige
Keltertrauben - und 4 pilzwiderstandsfähige Tafeltraubensorten entstanden. Diese Rebsorten sind mittlerweile als Vitis vinifera eingetragen und haben sich in den letzten Jahren weltweit verbreitet.

Ein großer Schritt ist sicherlich mit der Rebsorte Souvignier Gris gelungen. Die Cabernet Sauvignon x Bronner Kreuzung aus dem Jahr 1983 treibt mit den Burgundersorten aus und blüht einige Tage vor Grauburgunder. Der Traubenschluss erfolgt zum gleichen Termin wie bei Grauburgunder. Das Weichwerden und die Erntereife sind mit dem Weißburgunder vergleichbar. Souvignier Gris hat eine sehr hohe Pilzwiderstandsfähigkeit gegenüber Peronospora und eine hohe Pilzwiderstandsfähigkeit gegenüber Oidium. Die Traube ist mittel-groß und wenig kompakt. Diese Tatsache führt zu einer guten bis sehr guten Wirkung gegen Botrytis. Darüber hinaus sind die Beerenhäute sehr dick, so dass auch für tierische Schaderreger keine Möglichkeit besteht, die Beeren zu befallen. Aus der Traube können kräftige, stoffige Weine vergleichbar mit Grauburgunder produziert werden. Aus weinbaulicher Sicht ist Souvignier Gris eine sehr dankbare Sorte, die gut rankt und durch ihre längeren Internodien nicht zum Ineinanderwachsen der Trauben neigt.

\section{Neue Entwicklungen in der Rebenzüchtung WBI}

In Zusammenarbeit mit der INRA in Colmar (Frankreich) konnten in den Jahren 2004 bis 2006 französische Zuchtstämme aus dem Vitis muscadinia Genpool mit den pilzwiderstandsfähigen Freiburger Rebsorten gekreuzt werden. Die Zuchtstämme der INRA sind aus Rückkreuzungen mit den Rebsorten Cabernet Sauvignon, Grenache, Merlot und Aubun entstanden. Die Ergebnisse dieser Kreuzungen führten zu Zuchtstämmen, die neben einer weiteren Steigerung der Pilzwiderstandsfähigkeit eine deutlich spätere Reife erwarten lassen.

Der Zuchtstamm FR 628-2005r steht schon seit längerem im Focus der Untersuchungen. Er wurde in den letzten Jahren im Feld intensiv auf seinen weinbaulichen Wert untersucht. In der Weinausprägung konnte in den bisherigen Proben festgestellt werden, dass aus dieser Traube Weine hergestellt werden können, die in die fruchtige, körperreiche Richtung gehen.

Weitere Sämlinge wurden bisher aufgrund der vorhandenen Resistenzgene selektioniert. Diese Stöcke werden in den nächsten Jahren zu Zuchtstämmen aufgebaut und in Hinblick auf die weinbaulichen und oenologischen Eigenschaften untersucht.

\section{Literatur}

[1] A. Bouquet, J. Pauquet, A.F. Adam-Blondon, L. Torregrosa, D. Merdinoglu, S. WiedemannMerdinoglu, Bull. OIV 73, 445 (2000)

[2] C. Schneider, C. Onimus, E. Prado, V. Dumas, S. Wiedemann-Merdinoglu, M.A. Dorne, M.C. Lacombe, M.C. Piron, A. Umar-Faruk, E. Duchêne, P. Mestre, D. Merdinoglu, XII Int. Conf. Grapevine Breeding and Genetics (Bordeaux, France, 2018)

[3] V. Jörger, Der badischer Winzer 12, 27 (2008)

[4] H. Stücklin, Bereichsversammlung des Badischen Wienbauverbandes (2018) 